\title{
H comme Histoire : Hrotsvita, Hildegarde et Herrade, trois récits de fondation au féminin
}

\section{Laurence MOULINIER}

\section{(2) OpenEdition}

1 Journals

Édition électronique

URL : https://journals.openedition.org/clio/489

DOI : $10.4000 /$ clio.489

ISSN : 1777-5299

Éditeur

Belin

Édition imprimée

Date de publication : 1 novembre 1995

ISBN : 2-85816-283-2

ISSN : $1252-7017$

\section{Référence électronique}

Laurence MOULINIER, « H comme Histoire : Hrotsvita, Hildegarde et Herrade, trois récits de fondation au féminin », Clio. Histoire, femmes et sociétés [En ligne], 2 | 1995, mis en ligne le 01 janvier 2005, consulté le 29 avril 2022. URL : http://journals.openedition.org/clio/489 ; DOI : https://doi.org/ 10.4000/clio.489

Ce document a été généré automatiquement le 29 avril 2022.

Tous droits réservés 


\title{
H comme Histoire : Hrotsvita, Hildegarde et Herrade, trois récits de fondation au féminin
}

\author{
Laurence MOULINIER
}

1 On le sait de mieux en mieux aujourd'hui, certaines femmes du Moyen Age furent non seulement consommatrices, mais aussi productrices d'écriture, et les domaines dans lesquels elles laissèrent leur nom sont très variés, de la poésie à la médecine, en passant par l'art épistolaire, l'hagiographie, le théâtre, etc. L'histoire fut également une de leurs (pré)occupations et dès le Haut Moyen Age, la biographie de Radegonde composée vers l'an 600 par Baudonivie, moniale de Sainte-Croix de Poitiers, manifeste pleinement ce souci ${ }^{1}$ : comme l'a souligné Janet L. Nelson ${ }^{2}$, les femmes participaient de fait étroitement à la memoria, qu'il s'agisse de celle de la famille, de la gens, de la dynastie ou de la communauté dont elles faisaient partie.

2 Au-delà de l'initiale de leur prénom et d'une même appartenance à l'aire germanique, les trois femmes dont nous voulons parler ici ont eu en commun ce souci de l'Histoire et de leur histoire. Toutes trois religieuses, elles ont en effet tenu, chacune à sa manière, à livrer à la postérité le souvenir de l'abbaye à laquelle elles appartenaient. Hrotsvita de Gandersheim a composé au Xe siècle ses Primordia coenobii Gandeshemensis, Hildegarde de Bingen a écrit la Vita des saints patrons des deux monastères où elle vécut et, au XII siècle également, Herrade a immortalisé la fondation du Hohenbourg et la communauté qu'il abritait dans deux miniatures de son Hortus deliciarum.

3 Les différences entre ces trois femmes et leur mode d'expression respectif ne manquent évidemment pas. Si toutes trois étaient nonnes, il faut d'abord distinguer entre chanoinesses vivant selon la règle de saint Augustin et moniale bénédictine; deux d'entre elles, par ailleurs, furent abbesses et non seulement dirigèrent, mais aussi fondèrent des monastères, responsabilités que n'assuma pas la troisième; ajoutons enfin que deux d'entre elles jouissent aujourd'hui d'un statut et d'une célébrité d'auteurs à part entière tandis que la dernière, instigatrice d'une œuvre 
encyclopédique célèbre pour ses miniatures, fait plutôt figure de maître-d'œuvre, sinon de simple compilatrice et est somme toute encore relativement mal connue.

Autant de différences que nous tâcherons de mettre en évidence dans une présentation succincte de leur parcours et de leur apport respectif, pour nous concentrer ensuite sur ce qui rassemble ces femmes dans l'optique qui nous intéresse: qu'est-ce qui les a poussées à faire œuvre d'histoire locale, et surtout quels facteurs ont rendu possible, voire facilité leur entreprise?

5 Célèbre de longue date aux États-Unis au point d'avoir suscité la naissance d'un club portant son nom ("The Hroswitha Club», fondé en 1944 à New York par Robert Herndon Fife) et de se tailler la part du lion dans un roman pourtant hautement comique écrit dans les années 60 par John Kennedy Toole ${ }^{3}$, Hrotsvita de Gandersheim est en passe de le devenir en France : Patrick Corbet lui a fait une belle place dans son étude sur les saints ottoniens ${ }^{4}$, Ferruccio Bertini lui a consacré un essai dans Medioevo al femminile (traduit en français en 1991 sous le titre de La vie quotidienne des femmes au Moyen Age), et Monique Goullet a donné une édition critique et une nouvelle traduction de ses drames dans sa thèse, soutenue en 1993 et sur le point d'être publiée.

6 Hrotsvita, probablement née entre 930 et 935 et encore en vie en 973 , sans qu'on sache au juste la date de sa mort, n'était pas tout à fait une nonne, en tout cas certainement pas une nonne cloîtrée ${ }^{5}$. Si la règle bénédictine était observée à l'abbaye saxonne de Gandersheim où elle vécut, le régime était mixte. D'authentiques moniales - qui en 973 furent transférées à Notre-Dame de Gandersheim, la maison-mère devenant alors exclusivement un couvent de chanoinesses ${ }^{6}$ - y cohabitaient avec des chanoinesses, et ces dernières, qui n'étaient pas astreintes au vœu de pauvreté, non seulement pouvaient disposer de livres et de serviteurs et recevoir des hôtes, mais jouissaient plus généralement d'une relative autonomie ${ }^{7}$, y compris de mouvement : Hrotsvita aurait pu ainsi recevoir sa formation intellectuelle impeccable ${ }^{8}$ aux côtés de Bruno, frère du roi et archevêque de Cologne, lui-même très cultivé, sous la férule de Rathier de Vérone, présent à la cour en $952^{\circ}$. Autant de facteurs qui favorisèrent grandement l'activité littéraire de Hrotsvita, élève particulièrement douée de l'abbesse Richarde, qui lui enseigna sans doute les disciplines du quadrivium, et surtout de Gerberge (940-1001), fille du duc Henri de Bavière et nièce d'Otton I ${ }^{\text {er }}$. Succédant à Richarde à la tête de l'établissement, elle initia Hrotsvita au trivium et encouragea sa vocation littéraire en lui commandant notamment (la demande émanait également de Guillaume, archevêque de Mayence et fils naturel d'Otton) les Gesta Oddonis (965-968) ${ }^{10}$, récit des hauts faits d'Otton $\mathrm{I}^{\text {er }}$ et plus largement long poème épique à la gloire de la dynastie nouvellement installée qui devait s'éteindre en 1024.

7 L'établissement était étroitement lié aux Ottoniens. Il s'agissait d'une abbaye libre, responsable devant le souverain plutôt que devant l'Église, et à laquelle Otton I ${ }^{\text {er }}$ avait concédé en 947 le droit d'avoir sa cour, son armée et sa monnaie. Les membres de la dynastie saxonne faisaient donc figure de protecteurs de la collectivité religieuse tant sur le plan temporel que sur le plan spirituel, et, comme l'écrit $\mathrm{K}$. M. Wilson, après avoir évoqué les Ottoniens en tant que "secular rulers» dans ses Gesta, Hrotsvita s'attacha à les présenter comme des "religious rulers", "benefactors " dans ses Primordia coenobii Gandeshemensis.

8 Composée vers 970 , cette œuvre qui retrace les débuts de Gandersheim sur près de six cents hexamètres dactyliques (la dernière partie de la péroraison manque) est la dernière qui nous soit connue de Hrotsvita, et ses Primordia font d'elle, d'après M. 
Goullet, «le seul auteur féminin du haut Moyen Age à avoir écrit l'histoire de son abbaye "11, de sa fondation jusqu'à la mort de l'abbesse Christine en 919.

Fondé en 852, c'est le plus ancien établissement monastique institué par la noblesse saxonne, à savoir la famille comtale puis ducale des Liudolfing, et Hrotsvita fait rejaillir le mérite de cette fondation sur Otton lui-même, dans les premiers vers de son poème: "Voici que l'humble dévotion de mon faible talent brûle de chanter les origines de l'heureuse abbaye de Gandersheim, que fondèrent avec un zèle sans relâche les puissants ducs de Saxe, Liudolf le grand et son glorieux fils Otton, qui en acheva la construction $»^{12}$. Liudolf, dux orientalium saxonum, était l'époux d'Oda, elle-même fille d'un chef franc, Billung, et d'Aeda, remarquable par sa piété. De fait, c'est suite à une apparition de saint Jean-Baptiste à cette dévote femme que fut décidée la fondation : le saint ayant promis à Aeda que la fondation d'une abbaye de vierges garantirait à sa descendance la gloire suprême, Oda hâta l'exécution de ce vœu et plaça à la tête du nouvel établissement, d'abord provisoirement installé à Brunshausen, sa fille Hathumoda, qui le dirigea de 852 à 874 ; seules les filles nobles y étaient admises et deux autres filles des fondateurs succèderont à Hathumoda dans cette charge, Gerberge I de 874 à 896 et Christine de 896 à 919.

Comme les Gesta auxquels il fait d'ailleurs explicitement référence, cet écrit se présente donc comme une œuvre au service d'une stratégie hagiographique familiale. Les topoi n'y manquent pas, mais le rôle qui y est dévolu aux femmes est particulièrement original. On rangera ainsi parmi les passages obligés du récit de fondation le choix du lieu, désigné par un signe divin d'une lumière mystérieuse ou l'aspect inhospitalier de cet endroit, que Hrotsvita décrit comme une forêt véritable refuge de monstres ${ }^{13}$. Mais le relief que prennent les figures féminines dans ces Primordia (comme d'ailleurs dans les Gesta) est tout à fait remarquable: ce sont les femmes qui ont l'initiative du processus de fondation, Aeda, la Stammutter de la dynastie, n'est pas sans ressembler à Marie avec l'annonce qui lui est faite par saint Jean-Baptiste comme l'a souligné M. Goullet $^{14}$ et enfin Oda, sa fille, morte à 107 ans (longévité qui dut accroître son prestige selon P. Corbet) est la véritable héroïne de ces Primordia.

11 Le caractère presque exclusivement féminin de la sainteté ottonienne a été trop bien mis en évidence par P. Corbet pour qu'on y insiste. Soulignons plutôt la multiplicité des fonctions du récit de fondation que proposent les Primordia de Hrotsvita. Il a tout d'abord un rôle quasi mythologique, et tend à prouver l'origine divine du pouvoir impérial des Ottoniens par la mention de la promesse de saint Jean-Baptiste à Aeda. La fonction politique du récit en découle et, en montrant que le succès de la dynastie est la récompense de la piété d'Aeda, puis du soutien accordé par ses descendants au monastère, Hrotsvita légitime une nouvelle fois le règne des Ottoniens. Ce caractère partisan des Primordia expliquerait-il leur faible diffusion? Anne Lynn Haight estime à 5 seulement le nombre de manuscrits perdus de cette œuvre, dont il ne reste au demeurant aujourd'hui aucun manuscrit ancien ${ }^{15}$ et il n'est pas certain que ce texte ait été connu au-delà des toutes premières années du $\mathrm{XI}^{\mathrm{e}}$ siècle, et donc de l'extinction des Ottoniens. À moins que le fort ancrage local de ce récit n'ait guère intéressé les copistes? Sa fonction politique évidente se double en effet d'autres intentions : comme le rappelle M. Goullet, l'écriture des débuts de Gandersheim, établissement religieux, mais aussi seigneurie protégée par l'empereur, devait servir à affirmer l'identité de la communauté religieuse qui y était rassemblée, " en lui donnant des protecteurs sur le plan spirituel comme sur le plan temporel $»^{16}$. En les lui donnant ou en cherchant à les 
lui redonner? Il n'est pas exclu en effet que la composition des Primordia, et même des Gesta Oddonis, puisse aussi s'expliquer par la volonté de rappeler à leurs devoirs envers la communauté de Gandersheim les Ottoniens qui la délaissaient quelque peu au profit de leur nouvelle fondation de Quedlinburg, autre Familienkloster devant son origine aux efforts conjoints de Henri Ir et Mathilde - hypothèse que corroborerait le fait que Hrotsvita consacre une large place à Edith et Adélaïde, les deux femmes d'Otton Ir, contre trois vers seulement à Mathilde dans ses Gesta Oddonis.

12 Panégyrique, critique voilée ou récit des origines permettant à une religieuse d'affirmer l'identité de sa communauté et par là-même la sienne propre - Hrotsvita mentionne son propre nom dans sa version latine, clamor (hruot) validus (suid) ${ }^{17}$, pas moins de six fois dans l'ensemble de son œuvre - l'histoire des débuts du monastère de Gandersheim est donc susceptible de plus d'une lecture, mais il reste fondamental qu'il ait été écrit par une femme: "il témoigne de la conscience familiale et politique à laquelle avaient accédé les nobles saxonnes du haut Moyen Age $»^{18}$.

13 Hildegarde de Bingen (1098-1179) a entre autres en commun avec Hrotsvita une « voix forte » qu'elle n'eut pas peur de faire tonner à l'encontre des plus grands personnages de son temps, et peut-être même certains aspects de son œuvre ${ }^{19}$, mais elle s'en distingue radicalement par son état de moniale - puis abbesse - bénédictine et par sa qualité de visionnaire.

14 Ses nombreux autres dons lui valurent d'être un auteur particulièrement fécond, s'essayant à des domaines très divers: ses compositions musicales et ses écrits médicaux, notamment, lui valent aujourd'hui un regain de notoriété sans pareil, mais elle se fit également hagiographe et c'est sur cet aspect moins connu de son œuvre que nous voulons nous pencher.

15 Entrée comme oblate à huit ans au monastère du Disibodenberg, sur le Rhin, dans le diocèse de Mayence, elle reçut le voile à quinze ans des mains de l'évêque Otto de Bamberg ${ }^{20}$. Elle avait été confiée, pour son éducation, à la fille du comte de Spanheim, Jutta, qui y vivait en recluse. Lorsque celle-ci vint à mourir, en 1136, Hildegarde fut élue pour lui succéder comme magistra du Disibodenberg. Elle commença alors à prendre confiance en elle et osa s'ouvrir à un moine qui devait rester son confident jusqu'à sa mort en 1173, Volmar, des visions qu'elle avait depuis l'enfance. Elle avait commencé en 1141 à en coucher certaines par écrit dans ce qui allait devenir le Scivias, et lorsque cette œuvre, encore inachevée, eut reçu l'approbation du pape Eugène III lors d'un synode réuni à Trèves fin 1147/début 1148, Hildegarde, forte des encouragements du souverain pontife, put s'adonner sans crainte à une activité littéraire multiforme qui ne prit fin qu'à sa mort, trente ans plus tard. Or cette assurance qu'elle tirait de la bénédiction du plus haut représentant de Dieu sur terre, qui lui avait enjoint de livrer dorénavant aux hommes tout ce que lui dicterait l'Esprit saint, trouva également sa traduction dans la manière dont elle assuma sa charge de supérieure de monastère. Hildegarde s'enhardit et, sans d'ailleurs que personne, apparemment, n'élève la moindre protestation contre ses agissements, elle prit des initiatives dans des domaines jusqu'alors réservés aux hommes comme les séances d'exorcisme ou les tournées de prédication devant le clergé et le peuple de différentes villes d'Allemagne. La supérieure d'un monastère cloîtré partit donc quatre fois en mission, mais elle fit plus. Non contente d'égaler les hommes dans certaines de leurs activités, elle résolut de se séparer de la communauté masculine de son monastère d'origine. Comme le rapportent ses biographes ${ }^{21}$, elle avait reçu de Dieu, dans une vision, l'ordre d'aller fonder un 
nouveau cloître en un lieu alors à l'abandon, où étaient conservées les reliques d'un bienheureux ayant vécu à l'époque carolingienne, Rupert. La sécession ne se fit pas sans douleur, l'abbé du Disibodenberg, Cuno, voyant d'un très mauvais œil le départ de sa chère sœur visionnaire, désormais célèbre et donc source de prestige pour son monastère. Hildegarde tomba alors malade et dut s'aliter, frappée de cécité, selon elle, par un Dieu courroucé par son retard à s'exécuter. Elle réussit à convaincre l'abbé qu'il s'agissait là d'une punition divine, celui-ci finit par céder et Hildegarde partit avec dixhuit de ses sœurs, quittant l'oasis du Disibodenberg pour une Nature tout à fait inhospitalière et inculte où elle s'installa vers 1150. Abbesse d'une première communauté féminine, le Rupertsberg, elle en fonda une autre quinze ans plus tard de l'autre côté du Rhin (actuellement Rüdesheim-Eibingen), sous l'invocation de saint Gislebert. Elle s'y rendait régulièrement chaque semaine et, bien que fort occupée par différents travaux d'écriture, la femme de lettres restait une femme de tête qui se montrait particulièrement soucieuse d'assurer la bonne marche et la protection de ses deux fondations : en témoignent par exemple une charte de l'archevêque Arnold de Mayence qui, en 1158, confirme les droits reconnus au Rupertsberg (entre autres la libre élection de l'abbesse) par le précédent abbé du Disibodenberg, Cuno, désormais remplacé par Helinger ${ }^{22}$, ou encore le privilège impérial par lequel Frédéric $I^{\text {er }}$ Barberousse, en 1163, prend le Rupertsberg et ses possessions sous sa protection et réaffirme leur indépendance par rapport au Disibodenberg, dont l'abbé ne conserve que la cura animarum des sœurs du cenobium beati Roberti ${ }^{23}$. Rappelons que Hildegarde était d'origine noble. Elle fut entre autres l'amie de la tante du Hohenstaufen, et ce dernier la reçut personnellement en son palais d'Ingelheim en 1154. Les cloîtres fondés par Hildegarde étaient eux aussi réservés aux jeunes filles de la noblesse et elle sut agrandir le patrimoine de ses communautés en suscitant des donations en leur faveur de la part d'un certain nombre de nobles de la région.

Bien qu'ayant pris ses distances sur le plan juridique avec son monastère d'origine, elle resta en étroit contact avec les moines du Disibodenberg et ne se montra jamais oublieuse de son premier saint patron, bien au contraire. Elle mit son talent au service de la memoria de Disibod, un moine irlandais du VII ${ }^{\mathrm{e}}$ siècle fondateur du monastère où Hildegarde avait passé la première moitié de sa vie, et elle le célébra d'abord en composant en son honneur quatre chants liturgiques ${ }^{24}$. Puis, à la demande de l'abbé Helinger, elle s'attela en 1170 à la rédaction de sa Vita qui l'occupa trois ans durant, livrant ainsi à la postérité un document précieux tout d'abord par sa valeur historique puisque Hildegarde est, avec Hraban Maur, un des rares auteurs à avoir parlé de ce moine ${ }^{25}$.

Elle y développe donc des thèmes auxquels elle avait donné une expression poétique et souvent elliptique dans ses compositions liturgiques, comme celui de la montagne: Disibod y était appelé « montagne de l'esprit fermé ", mons clausae mentis ${ }^{26}$, en raison du lieu où il avait choisi de se fixer. Après une jeunesse pétrie d'humilité, d'amour de Dieu et de désir de pauvreté, il était venu d'Irlande jusqu'en Rhénanie avec trois disciples, Gillialdus, Clemens et Salustus ${ }^{27}$. Arrivé au bord du Glan, affluent de la Nahe, il avait avisé une montagne élevée et boisée, et dit à ses compagnons, touché par l'Esprit saint : "Ici sera mon repos ", hic requies mea erit. Sa vie de jeûne et d'austérité, sur le modèle de celles d'Antoine et Machaire ou encore de Paul qui voulait être « in silvis magis quam in villis $\aleph^{28}$, lui valut rapidement une renommée de sainteté et on lui construisit un oratoire. Le peuple, les pauvres et les malades affluent vers ce lieu appelé à l'origine "Studenheim », comme l'explique Hildegarde, à cause des taillis et des fourrés (studin 
en langue vulgaire) que l'on dut alors raser en l'honneur du saint homme. Où l'on retrouve le topos de la forêt et du débroussaillage lié à de nombreux autres récits de fondation, y compris, on l'a vu, celui concernant Gandersheim.

Les guérisons opérées par le saint tant de son vivant qu'après sa mort furent nombreuses, et il est frappant de reconnaitre dans le récit les mêmes catégories de malades que ceux que soigna Hildegarde d'après ses biographes: des aveugles, des boîteux, des infirmes, des possédés et ceux qui, en raison d'un désordre causé par les humeurs mauvaises, avaient perdu l'usage de leurs sens ${ }^{29}$. Que Hildegarde se soit délibérément reconnue dans la vie de ce père fondateur ou que ses propres biographes aient par la suite insisté sur les analogies de leurs parcours - tous deux moururent octogénaires, après avoir prédit leur mort - force est de constater que la Vita sancti Disibodi traduit à la fois la revendication d'une filiation spirituelle et la volonté de s'inscrire dans une histoire locale. La suite et fin du récit répond pour sa part aux règles du genre hagiographique : après la mort du saint, un monastère portant son nom (Mons sancti Disibodi, en allemand Disibodenberg) vit le jour, alors que Boniface était évêque de Mayence, autour de ses os et ses cendres, jusqu'alors conservés dans l'oratoire où Disibod avait été enseveli. Charlemagne accorda sa protection à l'établissement, mais à la mort de l'empereur, il connut le sort de nombreux autres monastères, à savoir une période de spoliations suivies d'un redressement.

C'est sans doute par le récit de la vie du bienheureux Rupert que Hildegarde contribua le plus à répandre une conception de la sainteté en accord avec ce que fut sa propre existence, et à se présenter comme la digne héritière du saint dont elle avait choisi le patronage pour sa première communauté féminine: plus encore que la Vita sancti Disibodi, la Vita sancti Ruperti, publiée pour la première fois par J. Köbel en 1524, est à la fois un pan d'histoire locale et un speculum réfléchissant sa propre image que l'abbesse tend à ses sœurs.

Fils d'une pieuse noble originaire de ce qui serait plus tard la Lotharingie, Bertha, et d'un « tyran » païen, Robold, Rupert vit le jour à l'époque de Charlemagne, in temporibus imperatoris Magni Caroli. Trompée par son mari qu'elle avait tenté en vain de convertir au christianisme, Bertha avait fait le vœu de ne pas se remarier si elle venait à être libérée de la tyrannie conjugale - et l'on ne peut s'empêcher de penser ici à l'indépendance que Hildegarde manifesta toute sa vie durant vis-à-vis du monde masculin. Bertha fut exaucée par la mort de son mari, et elle construisit alors une église sur la Nahe. C'est là que se trouvaient encore, à l'époque de Hildegarde, ses reliques et celles de son fils. Rupert, qui devait mourir à vingt ans, eut une enfance exemplaire, comme Hildegarde le clame aussi dans l'antienne qu'elle dédia à « l'enfance heureuse ", la felix pueritia de Rupert ${ }^{30}$ (notons au passage que dans ses chants, elle met pour ainsi dire à égalité ses deux principaux saints patrons : l'enfance de Disibod est également exaltée dans l'antienne " $O$ beata infantia $\|^{31}$, et Rupert se voit consacrer le même nombre de chants que Disibod). À sept ans, Rupert manifesta le désir d'apprendre les lettres, non pas tant pour devenir clerc que pour reprendre les fonctions de son père, duc de sa province, en se donnant toutefois pour mission la défense de l'Église. Chaste, refusant les plaisirs de ce monde et animé du souci de servir et de protéger l'Église, il mène une sainte existence auprès de sa mère qui le guide sur les voies de la piété, et il ne quittera sa colline de Bingen que pour se rendre à Rome. Revenu auprès de Bertha, que Hildegarde qualifie également de beata, Rupert songe un instant à abandonner son duché et toutes ses possessions pour se faire pélerin du Christ. Il restera, en fait, aux 
côtés de celle qui est son modèle et son soutien, sa mère (le rôle de cette dernière dans cette biographie est particulièrement important : lors de la maladie de Rupert, elle fait comme lui un songe prémonitoire, rêvant qu'on lui arrache une côte). Rupert consacre le reste de sa courte vie à l'édification d'églises et d'habitations. Lorsqu'il meurt, les fidèles affluent en masse vers l'oratoire qu'il avait fait construire avec sa mère et où on l'enterre. Sa sainteté trouve une première confirmation dans l'enthousiasme populaire qu'il suscite : « une foule immense venue de toute la région était présente $»^{32}$. Après sa mort, huit ans durant, les miracles et les signes se multiplient près de son tombeau. Bertha met alors l'ensemble de ses biens à la disposition de la congrégation de frères qui s'était établie là-bas, et, après vingt-cinq ans de vie pieuse faite de jeûnes, de prières et d'aumônes, elle s'éteint à son tour. Elle fut enterrée avec son fils et le mont SaintRupert, qui jouissait désormais d'une réputation de sainteté (locus in honore sanctitatis ${ }^{33}$ ) connut la paix jusqu'à l'arrivée des Normands, à la fin du IX siècle. Les habitants se transférèrent alors sur l'autre rive de la Nahe, emportant avec eux tout ce qu'ils pouvaient trouver, matériaux de construction compris, et le lieu fut dès lors voué à la désolation. Tous les biens de Rupert furent dispersés, sauf l'église qui, comme le note Hildegarde, existait encore à son époque, et quelques vignes dépendant de ladite église. C'est à Hildegarde qu'il appartint, sur ordre de Dieu, de faire revivre l'endroit en lui redonnant l'éclat spirituel qu'il avait trois siècles plus tôt. Elle s'y employa en restaurant tout d'abord l'église, puis en rachetant les vignes à l'évêque de Hildesheim Hermann et à son frère Bernard ${ }^{34}$, et en y installant sa communauté primitive de dixhuit moniales, qui devait prendre par la suite une ampleur telle que la fondation de Saint-Gislebert s'imposa comme une nécessité. Le récit hagiographique laissé par Hildegarde a donc au moins deux fonctions complémentaires. Il consacre l'action de l'abbesse en la présentant comme une continuatrice et il rappelle à ses lecteurs, surtout en fait à ses lectrices du Rupertsberg, aspirantes à la perfection, l'existence d'une tradition locale de sainteté initiée par saint Rupert et reprise par Hildegarde. Tradition locale à laquelle la personnalité et l'œuvre de l'abbesse donnèrent sans doute un plus ample rayonnement: si l'on en croit Trithemius dans sa Chronique de Hirsau, saint Bernard lui-même aurait demandé à Hildegarde une relique de saint Rupert ${ }^{35}$.

21 Venons-en pour finir à une autre abbesse, Herrade. On connaît mal ses origines, mais il paraît certain aujourd'hui que sa filiation avec les Landsberg d'Alsace est une invention du XVI $\mathrm{XIècle}^{\mathrm{e}}$ une idée d'abord suggérée par Jacques Wimpheling puis prise pour argent contant par Jérôme Gebwiler. Sa parenté spirituelle est, en revanche, plus aisée à cerner. Élève de l'abbesse Rélinde, qui dirigea le Hohenbourg de 1147 à 1163, elle lui succéda dans cette charge (en 1167 ou en 1176, les avis sont partagés). Nous sommes mieux renseignés sur ce que fut l'existence de Herrade à partir de son élection en tant qu'abbesse et jusqu'à sa mort, sans doute en 1195, et assurément après 1191. Son nom est attaché à l'Hortus deliciarum, œuvre monumentale (publiée pour la première fois entre 1879 et 1899 par les chanoines Straub et Keller ${ }^{36}$ ), commencée vers 1175 après de longues études préparatoires, dont la composition s'étala peut-être sur une trentaine d'années. Elle est célèbre avant tout pour ses très nombreuses miniatures, dont 254 sont aujourd'hui connues et 90 ont été perdues. Or l'inspiratrice de cette encyclopédie illustrée unique en son genre se doubla d'une abbesse extrêmement active, qui se fit même, comme Hildegarde, fondatrice d'établissements monastiques.

Les onze premières années de son abbatiat furent consacrées à des activités internes. Elle veilla à la restitution des biens qui avaient été distraits du patrimoine de l'abbaye, sollicita de nouvelles donations et œuvra à l'administration temporelle des domaines 
du Hohenbourg. Soucieuse d'exercer ses droits seigneuriaux, elle eut ainsi à cœur de définir le statut des habitants qui étaient sous sa dépendance directe ${ }^{37}$. Comme l'écrit $\mathrm{C}$. Bischof, « on devine une personnalité douée d'une inépuisable énergie et d'un sens aigu de l'organisation » à travers les chartes qui témoignent de son activité ${ }^{38}$. Le prologue de l'Hortus deliciarum lui-même, comme l'a suggéré J. Walter, commence, au reste, comme un diplôme : "Herrade, par la grâce de Dieu abbesse indigne du Hohenbourg... ». L'une des dernières miniatures du recueil - à laquelle nous allons nous intéresser plus en détail - représente la fondation du monastère et peut être lue comme une charte d'authenticité rappelant les origines et la destination du Hohenbourg.

En 1178 Herrade fit venir les Prémontrés d'Etival (Lorraine) à Saint-Gorgon, territoire alors abandonné, et remit entre les mains de l'abbé d'Etival, Warner, un livre symbolisant la donation. Deux ans plus tard, elle acheta avec un de ses ministériaux le domaine de Truttenhausen, au pied du château de Landsberg, pour y fonder un second établissement religieux et y installer des chanoines augustins réformés de Marbach (Haute-Alsace), communauté instituée en 1090 par Manegold de Lautenbach et important centre d'activités intellectuelles. Truttenhausen était une fondation plus importante que Saint-Gorgon (elle fut d'ailleurs confirmée en 1185 par une bulle de Lucius III) et comprenait une église, une ferme, un hôpital pour les pauvres et un hospice pour les pélerins et, bien sûr, un couvent. Le premier prieur fut un certain Volmar, arrivé avec 12 chanoines de Marbach. La précédente abbesse du Hohenbourg, Rélinde, avait conclu une confraternité de prières avec la communauté de Marbach, et les nouveaux arrivants s'engagèrent, de même que les religieux de Saint-Gorgon, à célébrer quotidiennement la messe au Hohenbourg. Le bénéfice que Herrade tira de ses deux fondations pour sa propre abbaye fut double, puisque ces chanoines qu'elle avait fait venir se partagèrent les offices religieux et les travaux d'érudition au Hohenbourg.

L'Hortus deliciarum doit sans doute, en effet, beaucoup à l'impulsion que le mouvement canonial donna à la vie spirituelle et intellectuelle, notamment à Marbach. Son école et son scriptorium étaient renommés - le Codex Guta Sintram en est issu - et l'on peut supposer que l'entreprise littéraire d'Herrade bénéficia d'une collaboration de ces chanoines. Si les sources dont son œuvre se nourrit ont pu lui être accessibles grâce à ses relations avec l'évêque et le chapitre de Strasbourg qui, selon J. Walter ${ }^{39}$, lui auraient ouvert les portes de la vieille bibliothèque de la cathédrale, il est probable aussi que les chanoines de Truttenhausen et de Saint-Gorgon l'ont aidée en lui procurant des manuscrits, en particulier d'œuvres contemporaines. L'Hortus deliciarum, gigantesque compilation vouée à l'enseignement des chanoinesses que Herrade avait sous sa direction, utilise en effet un nombre respectable d'écrits (plus de 1160 textes) dont certains émanaient d'auteurs contemporains tels Yves de Chartres, Marbode de Rennes, Rupert de Deutz, Honorius (qui, sans être nommé, fournit la matière d'environ 300 passages), saint Bernard (lui aussi utilisé sans être expressément cité), Pierre Lombard ou Pierre Comestor.

Par l'impressionnant ouvrage, un des premiers à être destiné à l'enseignement féminin, qu'elle légua à la postérité, Herrade dépassa donc Rélinde. Mais par l'activité qu'elle déploya en tant qu'abbesse, tenant encore en 1190, par exemple, à faire confirmer ses statuts par l'évêque Conrad II, l'élève se situait pleinement dans la lignée de sa maîtresse. La dernière miniature de l'Hortus, où Herrade est présentée comme Herrat Hohenburgensis abbatissa post Rilindam ordinata ac monitis et exemplis ejus instituta ( Herrade instituée abbesse du couvent de Hohenbourg après Rélinde, qui l'avait 
formée par ses leçons et ses exemples ») rappelle clairement cette dette. Le souvenir de celle qui l'avait formée lui tenait autant à cœur que l'histoire de son monastère, dont Rélinde n'était pas dissociable. Deux miniatures pleine page sont consacrées au Hohenbourg, la première évoque sa fondation et la seconde présente les portraits et les noms des chanoinesses et des converses qui y vivaient alors, Congregatio religiosa temporibus Relindis et Herradis abbatissarum in dei servitio in Hohenburg caritative adunata ( «a congrégation religieuse unie dans la charité pour le service de Dieu au monastère de Hohenbourg à l'époque des abbesses Rélinde et Herrade »).

L'institution du monastère de sainte Odile par le duc d'Alsace Adalric (alias Etichon ${ }^{40}$ ), à la fin du VII ${ }^{e}$ ou au début du VIII siècle, occupe la partie supérieure de l'avant-dernière miniature. La scène se passe sur la montagne même. Le duc y est représenté dans une position de respect. Il a ôté son manteau ducal doublé de fourrure et, agenouillé devant le Christ qui occupe le centre de l'image, il lui offre symboliquement le couvent en lui tendant un sceptre d'or. À côté, un texte explique ce geste : Sanctus Eticho dux qui et alio nomine dicitur Adalricus dotaliter offert Domino Jesu Christo et sanctae Mariae ac sancto Petro monasterium cum omnibus appendicis suis per se in monte hohenburc fundatum. La façade de l'église est encadrée à gauche par saint Pierre et la Vierge qui se font les intercesseurs du duc auprès du fils de Dieu et à droite par sainte Odile, la fille d'Etichon, et son patron privilégié, saint Jean-Baptiste. Adalric-Etichon avait confié la direction de l'établissement à sa fille, comme le donne à voir la partie intermédiaire de l'image. En vêtements d'apparat cette fois, le duc, assis dans un fauteuil, remet à Odile et à sa communauté (Congregatio sancta tempore sancte Odilie ad dei servitium in Hohenburc congregata) la clé qui symbolise son pouvoir.

Après la mort de sainte Odile, ses reliques devinrent, selon C. Bischof ${ }^{41}$, l'objet d'un pélerinage important assurant la prospérité et le renom du monastère, auquel les souverains carolingiens conférèrent l'immunité. Mais le Hohenbourg ne fut pas épargné par les troubles violents qui accompagnèrent en Alsace la Querelle des Investitures, et il tomba alors en décadence. Le duc de Souabe, Frédéric le Borgne, qui avait été écarté de l'Empire à la mort de Henri V en $1125^{42}$, s'attribua alors la charge d'avoué et détourna une partie du temporel de l'abbaye. Le redressement allait s'opérer grâce à son fils, Frédéric I ${ }^{\text {er }}$ Barberousse, qui apparaît comme le duc d'Alsace dès 1141. Le Hohenbourg était un lieu stratégique pour la défense des marches occidentales, et Barberousse voulait assurer des bases solides à la puissance des Hohenstaufen en Alsace, où il aimait à séjourner (entre 1152 et 1189 il s'y rendit à 13 reprises pour de grandes cérémonies ecclésiastiques et politiques, selon J. Walter ${ }^{43}$ ). Dès la mort de Frédéric le Borgne en 1147, Barberousse donna donc l'impulsion décisive à la restauration du monastère en plaçant à sa tête Rélinde, dont les écrivains postérieurs ont fait, à tort ou à raison selon E. et J. G. Rott ${ }^{44}$, sa parente. Un des signes de son intérêt pour le couvent de Sainte-Odile et son abbesse est par exemple la visite qu'il lui fit le 27 janvier 1153.

28 S'attachant à rétablir la discipline, Rélinde introduisit la règle de saint Augustin et donna un nouvel essor au monastère. Aussi fait-elle véritablement figure de seconde fondatrice dans la miniature qui nous occupe ici et qui lui rend hommage en la représentant au même étage et de la même taille que sainte Odile, à droite de l'image, et avec ce texte à côté d'elle: Rilinda venerabilis hohenburgensis ecclesiae abbatissa tempore suo ejusdem ecclesiae quaeque diruta diligenter restauravit et religionem divinam inibi pene destructam sapienter reformavit («Rélinde, vénérable abbesse du monastère de 
Hohenbourg, a soigneusement réparé de son temps tous les dommages du monastère et y a réformé avec sagesse l'esprit religieux alors presque perdu »). Comme le Christ, l'abbesse réformatrice exhorte ses sœurs à vivre saintement, et le message qu'elle leur adresse - son testament spirituel ? - se lit sur une croix, à sa droite («O cher troupeau, uni sous une loi céleste à l'abri de toute erreur, que celui que l'on appelle la montagne de Sion, qui sert de pont pour arriver à la patrie, qui est la source de tout bien, la voie et la lumière, te guide, que sa croix te protège !, etc. »).

Tout en bas de l'image, des branches de sapin assez sommairement dessinées évoquent les forêts du mont Sainte-Odile, comme sur la miniature suivante, qui complète la précédente et conclut l'ouvrage. Soixante bustes de moniales y sont alignés sur six étages (les treize converses occupent les étages inférieurs, après les jeunes nobles qui constituent le groupe des chanoinesses) : ils sont surmontés d'autant de prénoms et parfois d'une indication de leurs origines géographiques dont la variété reflète la renommée qu'avait reconquise le monastère grâce à l'action de Rélinde. L'image fond, au reste, dans une même temporalité son abbatiat et celui d'Herrade, qui s'affirme sa disciple. Comme Rélinde sur l'image de la fondation, Herrade figure en pied, à droite, et tenant dans ses mains une inscription à l'adresse de sa communauté, dans l'esprit de l'exhortation prêtée à sa maîtresse à la page précédente : 0 nivei flores dantes virtutis odores Semper divina pausantes in theoria Pulvere terreno contemto currite celo Quae nunc absconsum valeatis cernere sponsum ( $\mathrm{O}$ fleurs blanches comme la neige, qui répandez le parfum de vos vertus, méprisant la poussière terrestre, persistez dans la contemplation des choses célestes, et que votre course soit dirigée vers le ciel où vous verrez l'Époux encore caché à vos regards»). C'est sur cette illustration et ces mots rappelant sa filiation spirituelle qu'Herrade choisit de conclure son volumineux ouvrage pour l'édification de ses sœurs. L'image s'avérait un outil d'enseignement apte à immortaliser l'histoire d'une communauté, et par là même, à en resserrer les liens.

30 Au terme de ce parcours forcément rapide, nous trouvons pleinement confirmée par l'étude de nos trois auteurs l'idée, formulée par J. L. Nelson, selon laquelle les " historiennes sont à la fois exigées et permises par les conditions de leur époque »" Qu'elles aient fait œuvre de commande ou choisi spontanément de raconter l'histoire de leur communauté, nos trois femmes jouissaient en effet d'une liberté découlant de leurs rapports plus ou moins étroits avec le pouvoir alors en place. En outre, leur haute naissance et leur position stratégique dans leurs monastères respectifs leur assuraient un niveau culturel hors du commun. Qu'elles aient travaillé seules ou en équipe à la composition de leur récit de fondation, toutes trois bénéficièrent de contacts avec un entourage cultivé, et d'abord d'une solide formation dans les premières années de leur existence au couvent. Il est en effet remarquable que chacune d'entre elles ait été éduquée, pour ne pas dire initiée, par des femmes qui se sont avérées déterminantes pour la suite de leur existence et de leur " carrière ». Comme l'écrit encore J. L. Nelson pour le haut Moyen Age, "l'histoire n'était pas l'affaire de l'école, d'où les femmes étaient normalement exclues, mais de la cour ou de la domus $»^{46}$. Pour Hrotsvita comme pour Hildegarde et Herrade, la formation initiale qui allait permettre plus tard la réalisation et l'épanouissement de leur goût de l'histoire, leur a été dispensée par ces figures féminines dont elles se proclamèrent ensuite les disciples ou les héritières.

31 Le statut privilégié et la liberté dont elles bénéficiaient au sein de leur propre communauté se reflètent, sans doute, dans la variété et l'originalité des formes d'expression que «les trois $\mathrm{H}$ de l'aire germanique " choisirent pour délivrer leur 
message. La poésie métrique, comme l'hagiographie ou la miniature, s'avéraient aptes à traduire leur volonté de communiquer, voire de renforcer une conscience collective. C'était peut-être aussi une certaine façon de véhiculer leur fierté d'être femme. Les topoi d'humilité dont chacune d'elles ne manqua pas de parsemer son œuvre ne doivent pas nous tromper. Hrotsvita écrit en fait une véritable historia des femmes de la dynastie ottonienne, Herrade donne la supériorité aux personnages féminins dans son tableau de la fondation du couvent de Sainte-Odile où il faut noter que le Christ est présenté non comme le fils de Dieu, mais de la Vierge, Jhesus Christus Marie filius. Bien qu'ayant entrepris de raconter la vie de son saint patron Rupert, Hildegarde consacre des développements beaucoup plus nombreux à sa mère Bertha, dont elle fait à la fois une sainte femme et la véritable fondatrice du monastère qu'elle-même, Hildegarde, a été chargée par Dieu de redresser. Pour utiliser un vocabulaire en vogue, on pourrait donc dire qu'il y a du gender specific dans les trois documents auxquels nous nous sommes intéressés. Pour parler plus simplement, on peut conclure en disant que ce n'est pas dans le monde masculin (dont alternativement elles s'entourent, se font aider ou se passent), mais du côté des femmes que ces trois religieuses ont trouvé les modèles que leur récit de fondation tendait aussi à proposer, sinon à imposer.

\section{BIBLIOGRAPHIE}

BERTINI, F.

1989 « Rosvita, la poetessa », dans Medioevo al femminile, a cura di F. Bertini, Rome-Bari, Laterza : 63-95.

BISCHOF, C.

1979 « L'histoire » dans Herrad of Hohenbourg, Hortus deliciarum, R. Green dir., Londres-Leyde, Brill, 1979, 2 vol., vol. 1 : 9-17.

CORBET, P.

1986 Les saints ottoniens. Sainteté dynastique, sainteté royale et sainteté féminine autour de l'an mil, Sigmaringen.

DRONKE, $\mathrm{P}$.

1984 Women Writers of the Middle Ages, Cambridge.

GOULLET, M.

1993 Les drames de Hrotsvita de Gandersheim, édition critique avec introduction, traduction et notes, thèse de doctorat nouveau régime, Université de Metz.

1994 « Aspects du monachisme dans l'œuvre de Hrotsvita de Gandersheim », Revue Mabillon, nouvelle série 5 (t. 66) : 11-28.

HAIGHT, A. L. (éd.) 
1965 Hroswith of Gandersheim. Her Life, Times and Works, and a Comprehensive Bibliography, New York, The Hroswitha Club.

LEONARDI, C.

1989 « Baudonivia, la biografa », dans Medioevo al femminile, a cura di F. Bertini, Rome-Bari, Laterza : $31-40$.

NELSON, J. L.

1990 « Perceptions du pouvoir chez les historiennes du Haut Moyen Age », dans La femme au Moyen Age, M. Rouche et J. Heuclin éd., Publication de la ville de Maubeuge : 76-85.

PARISSE, M.

1983 Les nonnes au Moyen Age, Le Puy, C. Bonneton.

RICHEP.

1979 Ecoles et enseignement dans le haut Moyen Age, Paris.

ROTT, El. et J. G.

1945 Hortus deliciarum. Le «jardin des délices » de Herrade de Landsberg, un mansucrit alsacien à miniatures du XII ${ }^{e}$ siècle, Strasbourg, Oberlin.

VINAY, G.

1978 « Rosvita, una canonichessa ancora da scoprire ?», dans Alto Medioevo latino, Conversazioni e no (Esperienze 42), Naples : 483-554.

WALTER, J.

1952 Recueil de 50 planches, suivi du catalogue complet des 344 miniatures et du commentaire iconographique, Strasbourg-Paris.

WILSON, K. M.

1988 Hrotsvit of Gandersheim : the Ethics of Authorial Stance, Leyde, Brill.

WILSON, K. M. (éd.)

1987 Hrotsvit of Gandersheim : Rara avis in Saxonia?, The University of Georgia.

\section{NOTES}

1. Cf. Leonardi $1989: 36-40$.

2. Cf. Nelson 1990 : 76.

3. J. K. Toole († 1969), La conjuration des imbéciles, Paris, 1981 pour la traduction française.

4. Corbet 1986 : ch. III.

5. Cf. Dronke 1984 : 55-83.

6. Cf. Goullet $1993: 27$.

7. Parisse 1983 : 214.

8. Son drame Pafnutius montre par exemple clairement qu'elle n'ignorait ni la dialectique ni Boèce, cf. Riché $1979: 263$ et 275.

9. K. M. Wilson $1988: 150$. 
10. Voir la préface de l'œuvre : «Gerberge, illustre abbesse, vénérable pour sa haute vertu autant que pour l'éclat de sa royale noblesse, Hrotsvita de Gandersheim, la dernière des dernières qui servent sous votre commandement, vous salue comme une servante doit saluer sa maîtresse. Madame, vous dont l'esprit respendit d'innombrables rayons de sagesse, veuille votre Grâce ne pas répugner à la lecture d'un ouvrage dont elle sait qu'il fut écrit sur son ordre » (traduction de Monique Goullet, que je remercie chaudement de m'avoir communiqué ses travaux).

11. Goullet 1994 : 11 .

12. Traduction encore inédite de Monique Goullet, qui a eu la gentillesse de me la communiquer.

13. Goullet $1994: 14$ : « Ayant fait tailler les arbres et débroussailler l'endroit, il ordonna qu'on nettoyât la vallée ; et il rendit propre aux louanges divines ce lieu boisé, rempli de faunes et de monstres $»$.

14. Ibidem.

15. Haight $1965: 12$ ss.

16. Goullet $1994: 15$.

17. Le véritable sens de son nom ne fut découvert qu'en 1838 par Jacob Grimm ; on peut le comprendre comme « voix forte » mais aussi « solide renommée », fama valida; cf. K.

M. Wilson $1988: 145$.

18. Goullet $1994: 17$.

19. Des parallèles ont été menés entre leurs œuvres, toutes deux aussi riches que variées, par exemple entre le Dulcitius de la première et l'Ordo virutum de l'autre ; cf. Hozeski 1977 : 42-53.

20. Pour une biographie récente et en français de Hildegarde, on pourra se reporter à Régine Pernoud, 1994. Voir aussi la présentation de Bernard Gorceix, 1982.

21. Cf. Vita sanctae Hildegardis auctoribus Godefrido et Theodorico monachis, dans Sanctae Hildegardis abbatissae opera omnia, J.-P. Migne éd., PL 197, col. 91-130.

22. Cf. P. Acht, 1968, I : 417-418 (... spiritalem matrem secundum regulam beati Benedicti eligant libera electione).

23. Ibidem : 484-486 (... sub nostra tuicione suscipimus et iuxta instrumentum Arnoldi Maguntine sedis quondam venerabilis archiepiscopi imperiali privilegio tam praedictum locum quam ipsam abbatissam et sorores eius omnesque possessiones earum omni corroborationis munimine confirmamus).

24. Cf. Hildegarde de Bingen, Louanges, prés. et trad. L. Moulinier, Paris, La Différence, $1990: 52-58$ et 98.

25. Vita sancti Disibodi dans Sanctae Hildegardis abbatissae opera omnia, J.-P. Migne éd., PL 197, col. 1093-1116.

26. Cf. Louanges : 56-57.

27. Vita sancti Disibodi, col. 1101.

28. Ibidem, col. 1106.

29. Ibidem : illi qui de tempestate malorum humorum sensus suos amiserant.

30. Cf. Louanges : 98.

31. Ibidem.

32. Vita sancti Ruperti, dans Sanctae Hildegardis abbatissae opera omnia, J.-P. Migne éd., PL 197, col. 1083-1092, col. 1090.

33. Ibidem.

34. Ibidem, col. 1092. 
35. Cf. Chronicon insigne monasterii Hirsaugiensis, Ordinis S. Benedicti, per Ioannem Tritehemium, Bâle, 1559, ad a. 1148.

36. Hortus deliciarum, texte explicatif commencé par le chanoine A. Straub et achevé par le chanoine G. Keller, Strasbourg, 1899.

37. Bischof $1979: 11$.

38. Ibidem : 10.

39. Walter 1952.

40. Sa famille fonda également Murbach.

41. Bischof 1979.

42. C'est son frère Conrad qui fut élu empereur en 1138.

43. Walter 1952.

44. Rott 1945.

45. Nelson $1990: 83$.

46. Ibidem.

\section{RÉSUMÉS}

Un petit nombre de femmes-auteurs du Moyen Age se sont montrées particulièrement intéressées par l'Histoire, notamment locale, et, dans l'aire germanique, trois d'entre elles se distinguent par l'originalité de leur apport en ce domaine: Hrotsvita de Gandersheim au Xe siècle, et Hildegarde de Bingen et Herrade de Hohenbourg au XIIe. Toutes trois religieuses, elles ont livré à la postérité le récit de la fondation de leur monastère, l'une par le biais de la poésie métrique, la seconde via l'hagiographie et la dernière au moyen de l'image.

A small number of women in the Middle Ages were particularly interested in history - especially local history -, among which three in the germanic area singled themselves out by the originality of their contribution to this field: Hrotsvita of Gandersheim in the Xth century and in the XIIth century, Hildegard of Bingen and Herrad of Hohenbourg. The three of them were nuns, and they transmitted their accounts of the foundation of their respective convents : the first by means of metric poetry, the second through hagiography and the last in images.

\section{AUTEUR}

\section{LAURENCE MOULINIER}

Laurence MOULINIER est agrégée de Lettres modernes et docteur en Histoire. Elle a consacré sa thèse de doctorat, soutenue en 1994, à l'œuvre scientifique de Hildegarde de Bingen et a écrit différents articles sur l'histoire de l'environnement et de la médecine. Membre du comité de rédaction de la revue Médiévales depuis 1989, elle est actuellement Maître de Conférences d'Histoire médiévale à l'université de Poitiers. Elle a donné en 1990 la première traduction française des poésies complètes de Hildegarde, parue aux éditions de La Différence sous le titre de Louanges, et elle a tiré de sa thèse Le manuscrit perdu à Strasbourg : enquête sur l'œuvre scientifique de Hildegarde, Paris, Publications de la Sorbonne/Presses Universitaires de 
Vincennes, 1995. Adresse : Institut d'Histoire du Moyen-Age, Faculté des Sciences Anciennes, Université de Poitiers, 8 rue René Descartes, 86022 Poitiers Cedex. 